\title{
Editorial
}

\section{Antennas for Emerging 5G Systems}

\author{
Khalil H. Sayidmarie ${ }^{1 D},{ }^{1}$ Neil J. McEwan, ${ }^{2}$ Peter S. Excell, ${ }^{2,3}$ \\ Raed A. Abd-Alhameed, ${ }^{2}$ and Chan H. See ${ }^{4}$ \\ ${ }^{1}$ Department of Communication Engineering, Ninevah University, Mosul, Iraq \\ ${ }^{2}$ Department of Biomedical and Electronics Engineering, University of Bradford, Bradford, UK \\ ${ }^{3}$ Faculty of Art, Science and Technology, Wrexham Glyndwr University, Wrexham, UK \\ ${ }^{4}$ School of Engineering and the Built Environment, Edinburgh Napier University, Edinburgh, UK
}

Correspondence should be addressed to Khalil H. Sayidmarie; kh.sayidmarie@uoninevah.edu.iq

Received 28 May 2019; Accepted 30 May 2019; Published 1 July 2019

Copyright (C) 2019 Khalil H. Sayidmarie et al. This is an open access article distributed under the Creative Commons Attribution License, which permits unrestricted use, distribution, and reproduction in any medium, provided the original work is properly cited.

5G mobile communications technology will bring new experiences to industry and society, including higher data rates or greater capacity, higher bandwidth, increased security, and lower latency. These developments will create new opportunities for advancing society and businesses: $5 \mathrm{G}$ is going to make a significant change in how we live in this world.

The key enabling $5 \mathrm{G}$ technologies under development include novel multiple access strategies, massive MIMO, full digital beamforming or hybrid beamforming, ultra-dense networking, etc. The implementation of these high-level technologies will bring about new challenges for the designers of the physical infrastructure. These challenges undoubtedly include the antenna, but also the associated microwave systems and the characterisation of the radio propagation environment. In spite of very active current research on $5 \mathrm{G}$ antenna systems, including academic and industrial efforts, many challenges still need to be addressed, or they need better and more efficient solutions $[1,2]$.

5G will support significantly faster mobile broadband speeds and extensive mobile data usage as well as enabling the full potential of the Internet of Things [3]. From virtual reality and autonomous cars to the industrial Internet and smart cities, $5 \mathrm{G}$ will be at the heart of the future of communications. As well as conventional bands, 5G applications will have to utilize higher frequency bands in the millimetre wave region to enable wider bandwidth and higher data rates. The higher frequencies, wider bandwidths, and the need for beamforming, beam steering, and multiple beams are significant challenges to antenna designers [4]. Low-profile efficient antennas and antenna arrays to ensure reliable and interference-free communications are needed, but requirements for increased power, larger bandwidth, higher gain, and insensitivity to the presence of the human user further complicate the antenna and propagation aspects. This indicates the need for novel ideas and very innovative solutions in antenna design [5-7].

This special issue collects eight papers concerning various aspects of $5 \mathrm{G}$ antennas and their system applications. Two papers deal with modelling issues, while three papers are concerned with multiple-input multiple-output (MIMO) systems which are expected to be extensively used in the future 5G systems. Another paper considers an important issue of synthesizing appropriate radiation patterns of linear and planar antenna arrays of arbitrary geometry to provide the desired coverage reconfiguration. One of the papers deals with a single-layer dual-band reflectarray cell that is proposed for future $5 \mathrm{G}$ systems. Another paper discusses the direction finding problem in a background of unknown nonuniform noise using nested arrays, where a novel gridless direction finding method is proposed via the low-rank covariance matrix approximation. The following paragraphs give more elaboration about these subjects.

There are two papers dealing with the issue of modelling of the $5 \mathrm{G}$ systems. The paper by I. S. Batalha et al. provides an investigation into large-scale modelling of the propagation channel for frequencies above $6 \mathrm{GHz}$. The modeling concentrates on indoor environments, which will be a significant part of the implemented 5G systems. The paper considers a 
measurement investigation at $10 \mathrm{GHz}$ with directional horn antennas in a computer room and a corridor within an academic office building. The paper presents experimental data and channel modelling with various polarization scenarios such as copolarizations $\mathrm{H}-\mathrm{H}$ and $\mathrm{V}-\mathrm{V}$ and cross-polarization $\mathrm{V}$-H. Cases of both line-of-sight (LOS) and non-line-of-sight (NLOS) conditions were considered. The large-scale closein reference is sustained by comprehensive analysis, studying propagation mechanisms such as reflection and diffraction. The results demonstrated that the established model had an inferior standard deviation in relation to measured data, proving itself more significant to propagation in indoor environments.

The other paper by G. Liu et al. also considers modelling and deals with the interaction between the mobile user, who is in dynamic movement, and the wave propagation mechanism. The movements of a person holding a mobile handset can be considered as a stochastic process since the users can change the way they handle the mobile handset very frequently in a short time. These random actions of the hand-held mobile will change the antenna inclination angles resulting in various multipath propagation pathways which will lead to changes in the received signal intensity. Very few studies or conventional channel models have been performed to investigate these features. In this paper, the relationships between the statistical characteristics of the electric field and antenna inclination angles are investigated. They are modeled, based on a three-dimensional fast ray-tracing method which considers both the reflections and diffraction events: then the radiation patterns of an antenna with arbitrary inclination angels are deducted and included in the method. Two different conditions of the line-of-sight (LOS) and nonline-of-sight (NLOS) pathways in an indoor environment are discussed. Furthermore, based on a statistical analysis, a semiempirical probability density function of the antenna inclination angles is presented. Finally, a novel statistical channel model for stochastic antenna inclination angles is proposed and the ergodic channel capacity is analyzed.

Three other papers deal with an important application of $5 \mathrm{G}$ systems, deploying the transmitting and receiving antennas in MIMO configurations. The paper by J. J. Ding and J. Jiang investigates the application of hybrid precoding in massive multiple-input multiple-output (MIMO) systems. This technique can reduce the number of radio frequency (RF) chains. However, the consumed power is still very high owing to the use of a large-scale antenna array. In this paper, the authors propose a precoding scheme based on antenna selection technology: this precoding scheme greatly increases the energy efficiency (EE) of the system. Firstly, they derive an exact closed-form expression for energy efficiency. They further study the relationship between the number of transmit antennas and energy efficiency on the basis of the exact closed-form expression of efficiency. An optimal value was found. When the number of transmit antennas equals this value, the EE of the system can reach a maximum by using an appropriate hybrid precoding scheme. An antenna selection algorithm to select from the transmit antennas was then proposed, where the number of selected antennas equals the optimal value. Subsequently, design of the analog precoder based on a codebook to maximize the equivalent channel gain is presented, and then the EE can be improved by baseband digital precoding. The proposed precoding algorithm offers a compromise between spectral efficiency (SE) and EE in millimetre wave massive MIMO systems. Finally, simulation results are given to validate the theoretical analysis and show that a substantial EE gain can be obtained over the proposed precoding scheme without large performance loss.

Another paper deals with beamforming for millimetre wave massive MIMO-NOMA systems. The authors of the paper, J. Jiang et al., aim to provide a comprehensive scheme with limited feedback for downlink millimetre wave multiuser MIMO nonorthogonal multiple access (NOMA) systems. Based on the feedback of the best beam and the channel quality information (CQI) for this beam, the users are grouped into a cluster which has the same or coherent best beam and the maximal value of the CQIdifference. To further reduce the intercluster interference, only the candidate cluster can join the cluster set whose intercluster correlation with the existing clusters is lower than the threshold value. Based on the results of clustering, a hybrid beamforming strategy is designed. To improve the user experience, each cluster selects the best beam of the user with a higher guaranteed rate requirement as the analog beamforming vector. For digital beamforming, the weak user applies the block diagonalization algorithm based on the strong user's effective channel in order to reduce its intracluster interference. Finally, an intracluster power allocation algorithm is developed to maximize the power-difference in each cluster: this is beneficial to improve the successive interference cancellation (SIC) performance of the strong user. Simulation results are provided to show that the proposed MIMO-NOMA scheme offers a higher sum rate compared to the traditional orthogonal multiple access (OMA) schemes under practical conditions.

One of the papers considers the experimental evaluation of a MIMO-OFDM system with rateless space-time block code (STBC). A. H. Alqahtani et al. discuss the deployment of MIMO wireless technology in combination with orthogonal frequency division multiplexing (MIMO-OFDM) as an attractive technique for next-generation wireless systems. However, the performance of the wireless links is severely degraded because of the various channel impairments which cause a decoding failure and lead to packet loss at the receiver. One solution to such a problem is the rate-less space-time block code (RSTBC). The paper presents experimental results on the performance of a $2 \times 2$ MIMO-OFDM system with RSTBC as measured in a testbed implemented with a field programmable gate array (FPGA). The average bit error rate (BER) performance of the proposed scheme is evaluated experimentally, and the results agree closely with the simulation and analytical upper bound. The authors show that RSTBC can be implemented in real-world scenarios and will guarantee the reliability of loss-prone wireless channels.

The paper by G. Buttazzoni et al. considers the issue of antenna array reconfiguration by changing phase-only excitations to achieve Gaussian-shaped nulls. The proposed technique is intended for $5 \mathrm{G}$ applications. The paper presents 
a fast iterative method for the synthesis of linear and planar antenna arrays of arbitrary geometry that offer reconfigurable patterns for 5G applications. The method enables the generation of wide null regions shaped according to a Gaussian distribution: the results correlate with recent measurements on millimetre-wave angular dispersion. An experimental approach of phase-only control considers moving from the pattern provided by a uniformly excited array and iteratively modifying the phases of the excitations. This allows simplification in the feed network of the array as attenuators are not needed to vary the excitation magnitudes, therefore reducing the cost of realization of $5 \mathrm{G}$ base stations and mobile terminals. The proposed algorithm is based on the method of successive projections and relies on closed-form expressions for both the projectors and the null positions. It thus allows fast computation of the excitation phases at each iteration. Numerical examples compliant with $5 \mathrm{G}$ millimetre wave standards are presented to check the effectiveness of the proposed solution through scenarios that involve linear and concentric ring arrays.

In another paper, S. Costanzo et al. present a design for a single-layer dual-band reflectarray cell intended for future $5 \mathrm{G}$ system applications. A reflectarray unit cell operating at $28 / 38 \mathrm{GHz}$ is designed by adopting two pairs of miniaturized fractal patches, offering low losses $(<0.7 \mathrm{~dB})$ and full phase ranges (about $320^{\circ}$ ) at both operating frequencies. Dual-band operation achieved by utilizing fractal geometry is another application of this principle, which has been used for increasing the phase range at lower phase slope [8]. The proposed configuration offers very small interelement spacing and negligible mutual coupling effects between the two bands, thus enabling an independent phase tuning mechanism for both of the desired frequency bands. The compact cell demonstrated the reflectarray's abilities in achieving fixed scanned-beam and/or multibeam patterns, under the dual-band operation mode: such characteristics are beneficial and desirable in $5 \mathrm{G}$ systems. Full-wave numerical validations, performed on the synthesized reflectarray structures, confirmed the effectiveness of the designed dual-band configuration in achieving independent radiation patterns and good bandwidths, at the two designed frequencies.

Another paper considers yet another important issue in 5G systems: the reconstruction of the direction of the received waves. W. Tan and X. Feng address the direction finding problem in the background of unknown nonuniform noise with the nested array. A novel gridless direction finding method is proposed using the low-rank covariance matrix approximation. This approach is based on reweighted nuclear norm optimization. For the proposed method, the noise variance variable is eliminated by a linear transform, and then the covariance fitting criterion is utilized to determine the regularization parameter for ensuring robustness. The reconstructed low-rank covariance matrix is then obtained by iteratively reweighted nuclear norm optimization that imposes a nonconvex penalty. The search-free DOA estimation method is then utilized to perform the parameter estimation. The results of numerical simulations are given to verify the effectiveness of the proposed method. Moreover, results indicate that the proposed method gives a more accurate DOA estimation of both the nonuniform noise and off-grid cases compared to a state-of-the-art DOA estimation algorithm.

\section{Conflicts of Interest}

The editors declare that they have no conflicts of interest regarding the publication of this special issue.

\section{Acknowledgments}

The guest editorial team would like to express their gratitude to all the authors for their interest in selecting this special issue for their publications. The editors also wish to extend their appreciation to the anonymous reviewers for their careful reading of the twelve manuscripts submitted to this special issue and their valuable comments, suggestions, and following up of the revised versions of papers.

\author{
Khalil H. Sayidmarie \\ Neil J. McEwan \\ Peter S. Excell \\ Raed A. Abd-Alhameed \\ Chan H. See
}

\section{References}

[1] W. H. Chin, Z. Fan, and R. Haines, "Emerging technologies and research challenges for $5 \mathrm{G}$ wireless networks," IEEE Wireless Communications Magazine, vol. 21, no. 2, pp. 106-112, 2014.

[2] M. Shafi, A. F. Molisch, P. J. Smith et al., "5G: A tutorial overview of standards, trials, challenges, deployment, and practice," IEEE Journal on Selected Areas in Communications, vol. 35, no. 6, pp. 1201-1221, 2017.

[3] C. H. See, R. A. Abd-Alhameed, A. A. Atojoko, N. J. McEwan, and P. S. Excell, "Link budget maximization for a mobileband subsurface wireless sensor in challenging water utility environments," IEEE Transactions on Industrial Electronics, vol. 65, no. 1, pp. 616-625, 2018.

[4] W. Hong, Z. H. Jiang, C. Yu et al., "Multibeam antenna technologies for 5G wireless communications," IEEE Transactions on Antennas and Propagation, vol. 65, no. 12, pp. 6231-6249, 2017.

[5] N. O. Parchin, Y. I. Al-Yasir, A. H. Ali et al., "Eight-element dual-polarized MIMO slot antenna system for 5G smartphone applications," IEEE Access, vol. 7, pp. 15612-15622, 2019.

[6] W. Hong, K.-H. Baek, and S. Ko, "Millimeter-wave 5G antennas for smartphones: overview and experimental demonstration," IEEE Transactions on Antennas and Propagation, vol. 65, no. 12, pp. 6250-6261, 2017.

[7] M. Alibakhshikenari, M. Khalily, B. S. Virdee, C. H. See, R. A. Abd-Alhameed, and E. Limiti, "Mutual coupling suppression between two closely placed microstrip patches using EMbandgap metamaterial fractal loading," IEEE Access, vol. 7, pp. 23606-23614, 2019.

[8] K. H. Sayidmarie and M. E. Bialkowski, "Fractal unit cells of increased phasing range and low slopes for single-layer microstrip reflectarrays," IET Microwaves, Antennas \& Propagation, vol. 5, no. 11, pp. 1371-1379, 2011. 


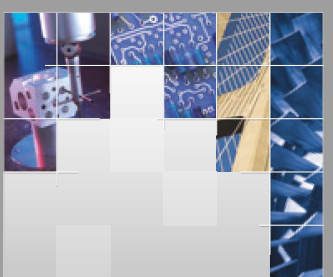

\section{Enfincering}
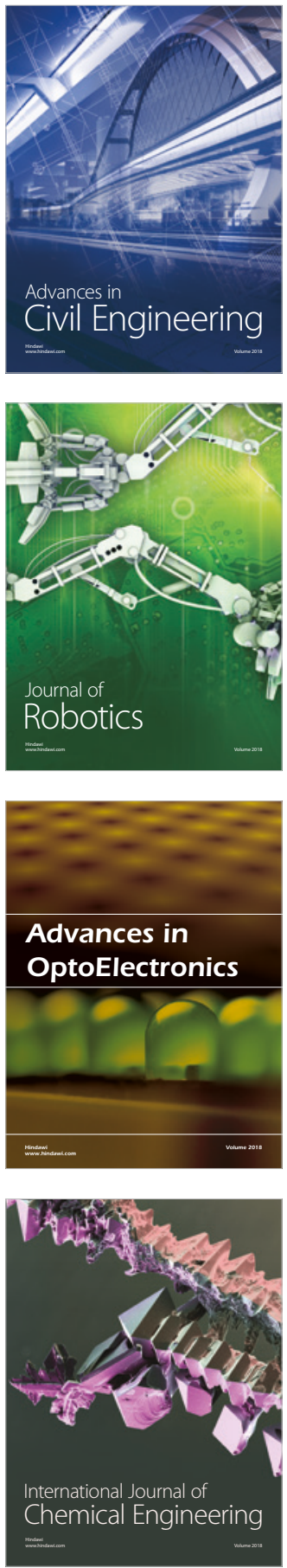

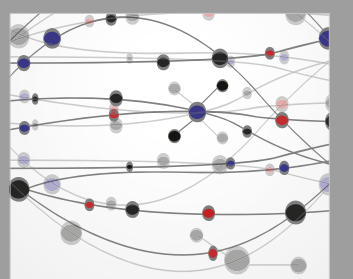

\section{Rotating \\ Machinery}

The Scientific World Journal

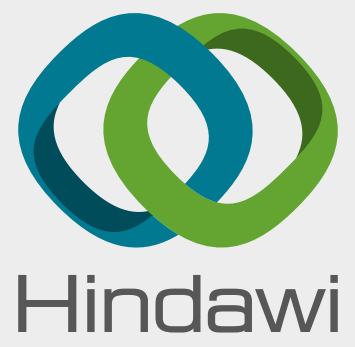

Submit your manuscripts at

www.hindawi.com
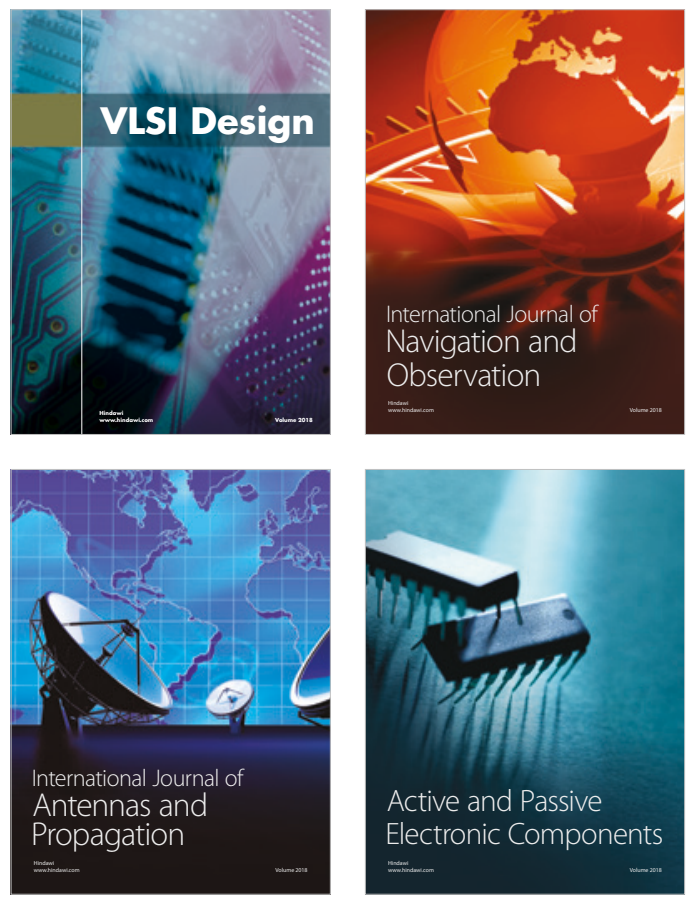
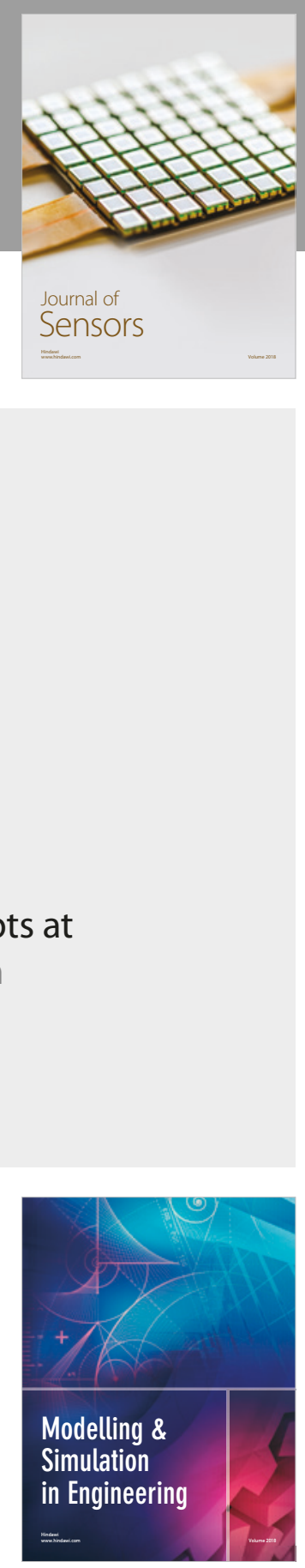

\section{Advances \\ Multimedia}
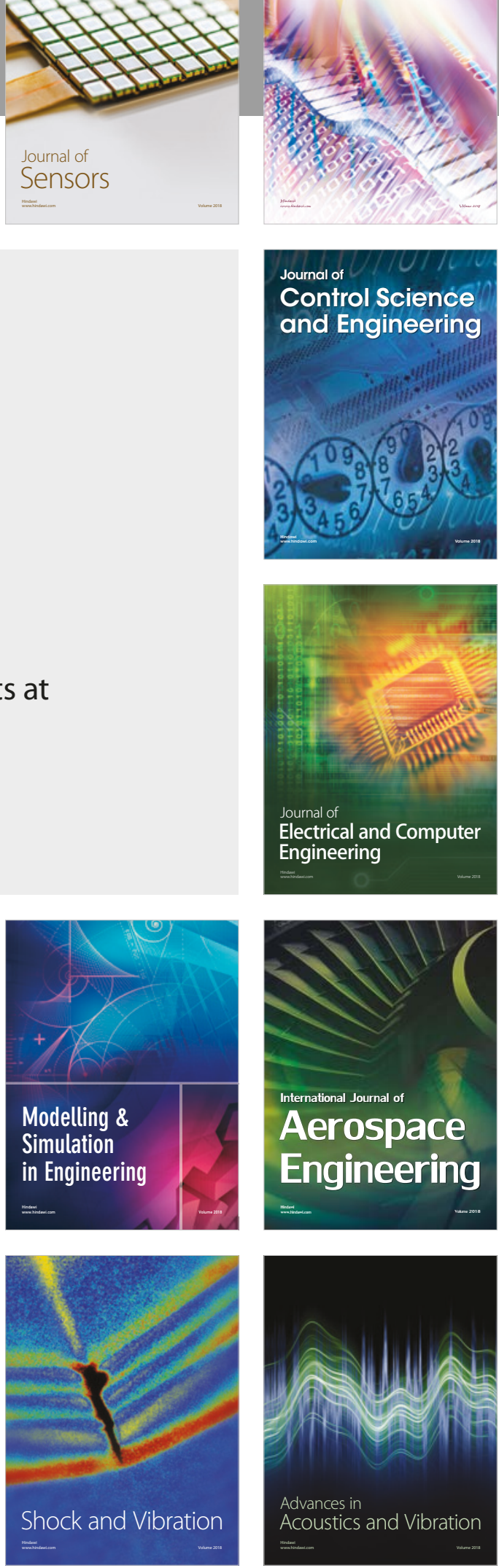